\title{
Apatinib suppresses the migration, invasion and angiogenesis of hepatocellular carcinoma cells by blocking VEGF and PI3K/AKT signaling pathways
}

\author{
JIFU SONG, ZHIBIN GUAN, CHAO SONG, MAOJIANG LI, ZHIWEI GAO and YONGLI ZHAO \\ Department of Radiotherapy, Qingdao Jiaozhou City Central Hospital, Jiaozhou, Qingdao 266300, P.R. China
}

Received August 11, 2020; Accepted March 23, 2021

DOI: $10.3892 / \mathrm{mmr} .2021 .12068$

\begin{abstract}
Hepatocellular carcinoma (HCC) is a commonly diagnosed malignancy worldwide with poor prognosis and high metastasis and recurrence rates. Although apatinib has been demonstrated to have potential antitumor activity in multiple solid tumors, the underlying mechanism of apatinib in HCC treatment remains to be elucidated. In the present study, apatinib were used to treat HCC cells transfected with or without VEGFR2 overexpression vectors. The proliferation of HCC cells was detected by MTT assay. The migration and invasion of HCC cells were detected by wound healing assay and Transwell assay. The ability of angiogenesis of HCC cells were detected by tube formation assay. The related protein expression levels were detected by western blotting. The present study aims to investigate the effect and potential mechanism of apatinib on the migration, invasion and angiogenesis of HCC cells. It was found that apatinib treatment significantly inhibited the proliferation, migration and invasion of Hep3b cells and suppressed angiogenesis in HUVECs. In addition, apatinib inhibited the epithelial-mesenchymal transition of Hep3b cells by increasing the expression of the epithelial hallmarks E-cadherin and $\alpha$-catenin and decreased the expression of the mesenchymal hallmarks $\mathrm{N}$-cadherin and vimentin. These effects were associated with the downregulation of VEGF and VEGFR2 and suppression of the PI3K/AKT signaling pathway. Thus, apatinib inhibited cell migration, invasion and angiogenesis by blocking the VEGF and PI3K/AKT pathways, supporting an effective therapeutic strategy in the treatment of HCC.
\end{abstract}

\section{Introduction}

Hepatocellular carcinoma (HCC) is one of the severest types of human cancer and is the third-leading cause of cancer-related mortality worldwide (1). Although the treatment of HCC has

Correspondence to: Dr Yongli Zhao, Department of Radiotherapy, Qingdao Jiaozhou City Central Hospital, 29 Xuzhou Road, Jiaozhou, Qingdao 266300, P.R. China

E-mail: yonglizhao0807@163.com

Key words: hepatocellular carcinoma, apatinib, VEGF, PI3K/AKT improved, the long-term prognosis of patients with $\mathrm{HCC}$ is still poor, mainly due to the advanced stages of the disease at the time of diagnosis and its high metastasis and recurrence rates (2). Therefore, potential drugs with fewer side effects that could significantly inhibit the growth of existing tumors and prevent cancer cell metastasis, invasion and angiogenesis are required for treating $\mathrm{HCC}(3)$.

The epithelial-mesenchymal transition (EMT) is an important mechanism for epithelial-derived tumor cells to become malignant and acquire an invasive phenotype $(4,5)$. It is accompanied by the downregulation of the epithelial marker E-cadherin and the upregulation of the mesenchymal markers vimentin and $\mathrm{N}$-cadherin (6,7). Previous studies have identified that metastatic cancer cells induce EMT by modulating their cell shape or adhesive properties, thereby affecting cell adhesion and migration $(8,9)$. Therefore, EMT serves an important role in tumorigenesis, invasion and metastasis.

VEGF is a signal protein released by epithelial cells and serves an important role in cell proliferation and neovascularization in several cancers, including HCC (10). In addition to the well-known effects of VEGF on angiogenesis, VEGF signaling serves an important role in promoting the proliferation and inhibiting the apoptosis in tumor cells (11). In HCC, multivariate analyses suggests that only a strong VEGF expression in tissue is significantly associated with metastatic recurrence (12). VEGF can stimulate the activation of the VEGFR2-dependent mTOR pathway to promote angiogenesis in lung cancer cells (13). Furthermore, there is evidence that the proliferation, migration, invasion and adhesion of non-small cell lung cancer (NSCLC) cells are significantly inhibited by blocking the VEGF/VEGFR2 pathway (11).

Apatinib is a tyrosine kinase inhibitor that selectively inhibits VEGFR2, resulting in the blocking of the intracellular VEGF signaling pathway (14). As a new oral antiangiogenic agent, apatinib has shown encouraging clinical results in treating various solid tumors (15-17). Apatinib has been identified as the only effective drug for patients with terminal gastric cancer without chemotherapy indications in a phase III clinical trial (18). In a phase II clinical trial, apatinib monotherapy is effective and safe in advanced HCC (19). However, the underlying mechanism of apatinib against $\mathrm{HCC}$ remains to be elucidated.

The present study explored the effect and potential mechanism of apatinib in HCC cell migration, invasion and 
angiogenesis using the Hep3b cell line. It was found that apatinib reduced the proliferation, migration and invasion of Hep3b cells by regulating VEGF and PI3K-AKT signaling pathways.

\section{Materials and methods}

Cell culture and reagents. The human HCC cell line Hep3b was purchased from the Cell Resource Center of Shanghai Institutes for Biological Sciences, Chinese Academy of Sciences and cultured in RPMI-1640 (Gibco; Thermo Fisher Scientific, Inc.) supplemented with $10 \%$ fetal bovine serum (FBS; Gibco; Thermo Fisher Scientific, Inc.), $100 \mathrm{U} / \mathrm{ml}$ penicillin and $100 \mu \mathrm{g} / \mathrm{ml}$ streptomycin. Human umbilical vein endothelial cells (HUVECs) were purchased from the American Type Culture Collection and maintained in Dulbecco's modified Eagle's medium (DMEM)/F12 (Gibco; Thermo Fisher Scientific, Inc.) containing 10\% FBS supplemented with $100 \mathrm{U} / \mathrm{ml}$ penicillin and $100 \mu \mathrm{g} / \mathrm{ml}$ streptomycin. All cells were cultured in a humidified chamber at $37^{\circ} \mathrm{C}$ with $5 \% \mathrm{CO}_{2}$. Apatinib was purchased from Jiangsu Hengrui Medicine Co., Ltd. and was dissolved in dimethyl sulfoxide (DMSO).

Cell transfection. VEGFR2 overexpression vector and empty vector $(\mathrm{EV})$ were purchased from VectorBuilder. VEGFR2 were amplified by using the sense primer 5'-TGTCGTTGTAGGGTAT AGGATTTATGAT-3' and the anti-sense primer 5'-ATACTT GTCGTCTGATTCTCCAGGTTTC-3'. Following the manufacturer's instructions for Lipofectamine ${ }^{\circledR} 2000$ (Invitrogen; Thermo Fisher Scientific, Inc.), Hep3b cells at $80 \%$ confluence were transfected with $1 \mu \mathrm{g} / \mathrm{ml}$ VEGFR2 expression vector or EV. After incubated at $37^{\circ} \mathrm{C}$ with $5 \% \mathrm{CO}_{2}$ for $24 \mathrm{~h}$ for $24 \mathrm{~h}$, cells successfully transfected with the vectors were used for subsequent experiments; the transfection efficiency was verified by fluorescence microscopy (Fig. S1).

MTT assay. After Hep3b cells were cultured overnight in 96-well plates $\left(1 \times 10^{5}\right.$ cells/well), the following three treatment conditions were set: i) Cells treated with 0, 20, 40 and $60 \mu \mathrm{M}$ apatinib for $24 \mathrm{~h}$; ii) cells treated with $40 \mu \mathrm{M}$ apatinib for 12, 24, 48 and $72 \mathrm{~h}$; and iii) cells transfected with VEGF overexpression vector or $\mathrm{EV}$ for $2 \mathrm{~h}$ following treatment with $40 \mu \mathrm{M}$ apatinib for $24 \mathrm{~h}$. Following culture for another $24 \mathrm{~h}$, $10 \mathrm{mg} / \mathrm{ml}$ MTT was added into each well and incubated for another $4 \mathrm{~h}$. Cells were centrifuged at $1,000 \mathrm{x} \mathrm{g}$ for $5 \mathrm{~min}$ at room temperature. DMSO (100 $\mu \mathrm{l})$ was added into each well and incubated for $30 \mathrm{~min}$ to dissolve the formazan product. The absorbance value of each well was measured at a $490 \mathrm{~nm}$ wavelength.

Transwell assay. Hep3b cells were resuspended in RPMI-1640 supplemented with $1 \%$ FBS and $1 \times 10^{4}$ Hep3b cells were seeded into the top chamber and treated under two conditions: i) Cells treated with $40 \mu \mathrm{M}$ apatinib for $24 \mathrm{~h}$; and ii) cells transfected with VEGF overexpression vector or EV for $2 \mathrm{~h}$ following treatment with $40 \mu \mathrm{M}$ apatinib for $24 \mathrm{~h}$. Cells in the upper chamber were then gently removed and the invaded cells were collected and fixed for 30 min using $4 \%$ paraformaldehyde, stained with $0.1 \%$ crystal violet for $30 \mathrm{~min}$ at room temperature and washed three times with PBS. The number of cells was then counted under an optical microscope (x100 magnification; Olympus Corporation).

Wound healing assay. Hep3b cells were seeded into 6-well plates at $2 \times 10^{5}$ cells/well and two treatment conditions were set: i) Cells treated with $40 \mu \mathrm{M}$ apatinib for $24 \mathrm{~h}$; and ii) cells transfected with VEGF overexpression vector or EV for $2 \mathrm{~h}$ following treatment with $40 \mu \mathrm{M}$ apatinib for $24 \mathrm{~h}$. The wound gap on the cell monolayer was created using a $200 \mu$ l pipette tip and cultured in serum-free RPMI-1640. An optical microscope at X100 magnification was used for imaging and the migration of cells was observed at $24 \mathrm{~h}$ after wound scratching.

Matrigel in vitro HUVEC tube formation assay. The conditioned media $(\mathrm{CM})$ of $\mathrm{Hep} 3 \mathrm{~b}$ cells were collected and stored at $-80^{\circ} \mathrm{C}$. HUVECs were trypsinised and seeded $\left(5.0 \times 10^{4}\right.$ cells per well) into Matrigel-coated wells (coated with Matrigel at $4^{\circ} \mathrm{C}$, then incubated for $30 \mathrm{~min}$ at $37^{\circ} \mathrm{C}$ ) with $250 \mu \mathrm{l}$ of $\mathrm{CM}$ from Hep3b cells. Following incubation at $37^{\circ} \mathrm{C}$ for $24 \mathrm{~h}$, six different fields were randomly chosen in each well and images were captured.

Western blotting analysis. Hep3b cells were washed in PBS and lysed using the protein extraction reagent RIPA (Invitrogen; Thermo Fisher Scientific, Inc.). The concentration of proteins was measured by BCA kit (cat. no. ab102536; Abcam). Equivalent amounts of proteins ( $30 \mu \mathrm{g})$ from each sample were electrophoresed on SDS-polyacrylamide gel (SDS-PAGE) and transferred onto a polyvinylidene fluoride membrane, blocked in $4 \%$ skim milk for $2 \mathrm{~h}$ at room temperature and incubated with the following specific primary antibodies: E-cadherin antibody (ab219332 1:1,000; Abcam), $\alpha$-catenin antibody (ab51032 1:2,000; Abcam), N-cadherin (ab76011, 1:5000 dilution, Abcam), vimentin (ab92547 1:1,000; Abcam), p-PI3K (ab182651 1:1,000; Abcam), PI3K (ab227204 1:1,000; Abcam), p-AKT (ab38449, 1:500 dilution, Abcam), AKT (ab18785 1:1,000; Abcam), VEGF (ab214424 1:1,000; Abcam), VEGFR2 (ab221679 1:1,000; Abcam), Snail (ab53519 1:1,000; Abcam), Slug (ab27568 1:1,000; Abcam) and MPP9 (ab38898 1:1,000; Abcam) overnight at $4^{\circ} \mathrm{C}$. $\beta$-actin (ab8277 1:1,000; Abcam) was used as internal reference. Then, the membranes were incubated in HRP-linked goat anti-rabbit IgG secondary antibody (ab97051; 1:10,000; Abcam) for $2 \mathrm{~h}$ at room temperature. Immunoreactivity was visualized by a colorimetric reaction using an ECL substrate buffer (EMD Millipore) and membranes were scanned with Gel Doz EZ imager (Bio-Rad Laboratories, Inc.).

Statistical analysis. Data are shown as the mean \pm standard deviation. Statistical analysis was performed using Tukey's post hoc test for one-way analysis of variance using the SPSS 16.0 software package (SPSS, Inc.). $\mathrm{P}<0.05$ was considered to indicate a statistically significant difference.

\section{Results}

Apatinib inhibits the proliferation of Hep $3 b$ cells. Compared with the control group, 10, 20, 40 and $60 \mu \mathrm{M}$ apatinib treatment significantly reduced the proliferation of Hep3b cells (Fig. 1A). Treatment with $40 \mu \mathrm{M}$ apatinib for $24 \mathrm{~h}$ inhibited the proliferation of Hep3b cells and a greater decrease in 

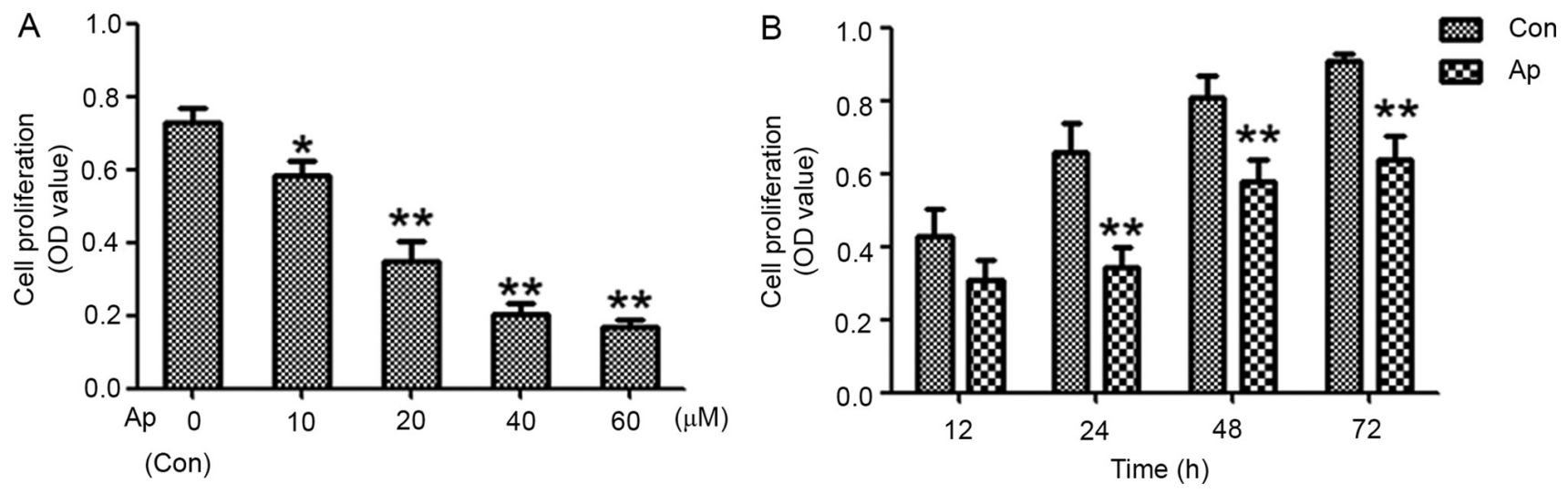

Figure 1. Apatinib inhibited the proliferation of Hep3b cells. (A) Hep3b cells were incubated for $24 \mathrm{~h}$ in the presence of various concentrations of apatinib. (B) Hep3b cells were incubated in the absence or presence of $40 \mu \mathrm{M}$ apatinib for the indicated time interval. Values are shown as mean \pm standard deviation ( $\mathrm{n}=3$ ); $\mathrm{P}<0.05$, *** $\mathrm{P}<0.01$ vs. Con group. Con, control; OD, optical density; Ap, apatinib.

cell proliferation was observed upon treatment with apatinib for 48 and $72 \mathrm{~h}$ (Fig. 1B). These results indicated that apatinib decreases the proliferation of Hep3b cells in a dose- and time-dependent manner.

Apatinib inhibits the migration and invasion of Hep3b cells. Previous studies have revealed that apatinib reduces tumor cell metastasis by inhibiting EMT $(20,21)$. The present study investigated the metastatic ability of Hep3b cells following treatment with $40 \mu \mathrm{M}$ apatinib using the Transwell and wound healing assays. It was found that apatinib treatment was sufficient to reduce invasion of Hep3b cells (Fig. 2A) and inhibit migration (Fig. 2B). EMT-related protein levels in Hep3b cells treated with or without $40 \mu \mathrm{M}$ apatinib were detected by western blotting. The results showed that apatinib significantly induced protein expression of E-cadherin and $\alpha$-catenin and reduced protein expression of $\mathrm{N}$-cadherin, vimentin, Snail, Slug and MMP9 in Hep3b cells (Fig. 2C). The PI3K/Akt pathway activation is known to be involved in tumor cell invasion and metastasis in response to various growth factors, including EMT $(22,23)$. In the present study, the treatment of $40 \mu \mathrm{M}$ apatinib significantly inhibited the activation of the PI3K/AKT pathway (Fig. 2D). These data demonstrated that apatinib inhibited migration and invasion by suppressing the PI3K/AKT pathway-dependent EMT in Hep3b cells.

Apatinib inhibits the angiogenesis of HUVEC cells. Next, it was determined whether the conditioned medium from Hep3b cells could regulate tube formation in HUVECs. After $24 \mathrm{~h}$ of incubation, CM from Hep3b cells treated with apatinib has decreased the extent of tube formation by HUVECs compared with the control group (Fig. 3A). To further elucidate the underlying molecular mechanism of apatinib on angiogenesis, protein expression levels of VEGF and VEGFR2 were determined upon cell treatment. It was found that treatment of Hep3b cells with $40 \mu \mathrm{M}$ apatinib led to decreases in VEGF and VEGFR2 protein levels (Fig. 3B).

VEGFR2 overexpression abolishes the inhibitory effect of apatinib on Hep3b cell migration and invasion. Knowing that apatinib inhibited expression of VEGF and VEGFR2 and signaling activity in Hep3b cells, the role of VEGF signaling in apatinib-treated Hep3b cells was next determined. VEGFR2 overexpression significantly counteracted the inhibitory effects of apatinib on the proliferation, migration and invasion of Hep3b cells (Fig. 4A-C). VEGFR2 overexpression significantly induced EMT in apatinib-treated Hep3b cells via the downregulation of E-cadherin and $\alpha$-catenin and upregulation of N-cadherin, vimentin, Snail, Slug and MMP9 (Fig. 4D). In addition, VEGFR2 overexpression significantly induced the activation of the PI3K/AKT pathway by increasing the levels of phosphorylated PI3K and AKT in Hep3b cells (Fig. 4E).

VEGFR2 overexpression abolishes the inhibitory effect of apatinib on the angiogenesis of HUVEC cells. After $24 \mathrm{~h}$ of incubation, CM from Hep3b cells transfected with VEGFR2 overexpression vector combined with apatinib treatment increased the extent of tube formation by HUVECs compared the Ap group (Fig. 5A). The protein expression levels of VEGF and VEGFR2 in Hep3b cells transfected with the VEGF overexpression vector combined with apatinib treatment were higher than the Ap group (Fig. 5B).

\section{Discussion}

Liver cancer is one of the three most lethal cancers. Although patients with $\mathrm{HCC}$ exhibit increased survival rates following curative resection, the prognosis of patients with $\mathrm{HCC}$ remains poor due to tumor metastasis and invasiveness (24). Apatinib is a highly selective inhibitor of multiple tyrosine kinases and one of the latest agents with encouraging preclinical and clinical data in treating solid tumors (25). Previous studies have shown that apatinib treatment leads to apoptosis and autophagy and inhibits EMT and metastasis in osteosarcoma cells $(26,27)$. The present study found that apatinib significantly inhibited the proliferation, invasion and metastasis of Hep3b cells. This was consistent with a previous study on cholangiocarcinoma, which found that apatinib inhibits cellular migration and invasion via the PI3K/AKT pathway (28). The PI3K/AKT signaling pathway serves an important role in regulating tumor growth, angiogenesis, apoptosis, invasion and metastasis (29). Abnormal activation of the PI3K/Akt/mTOR signaling pathway occurs in $\sim 45 \%$ of HCC cases and is associated with the poor prognosis in patients with HCC through related 

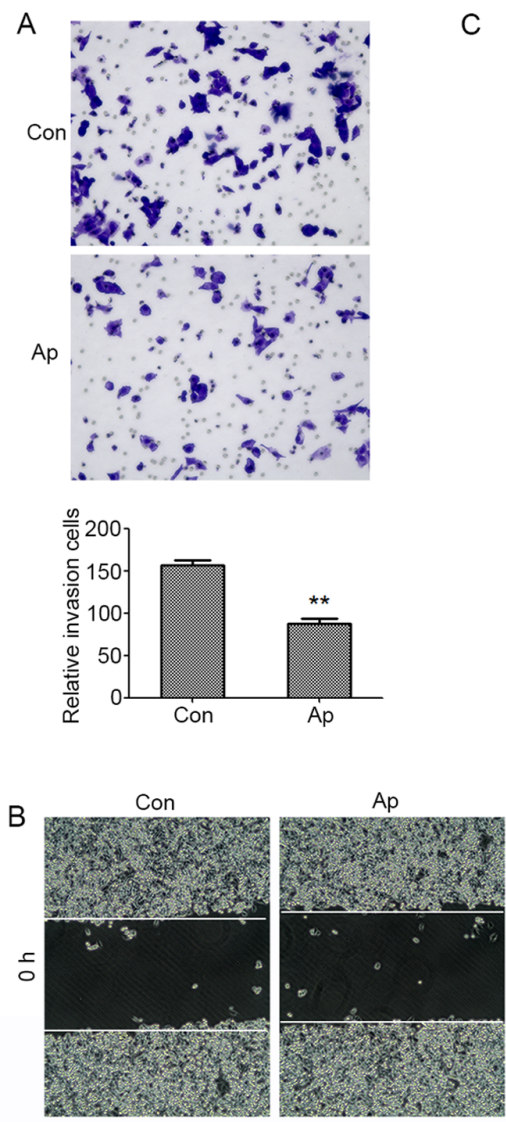

C
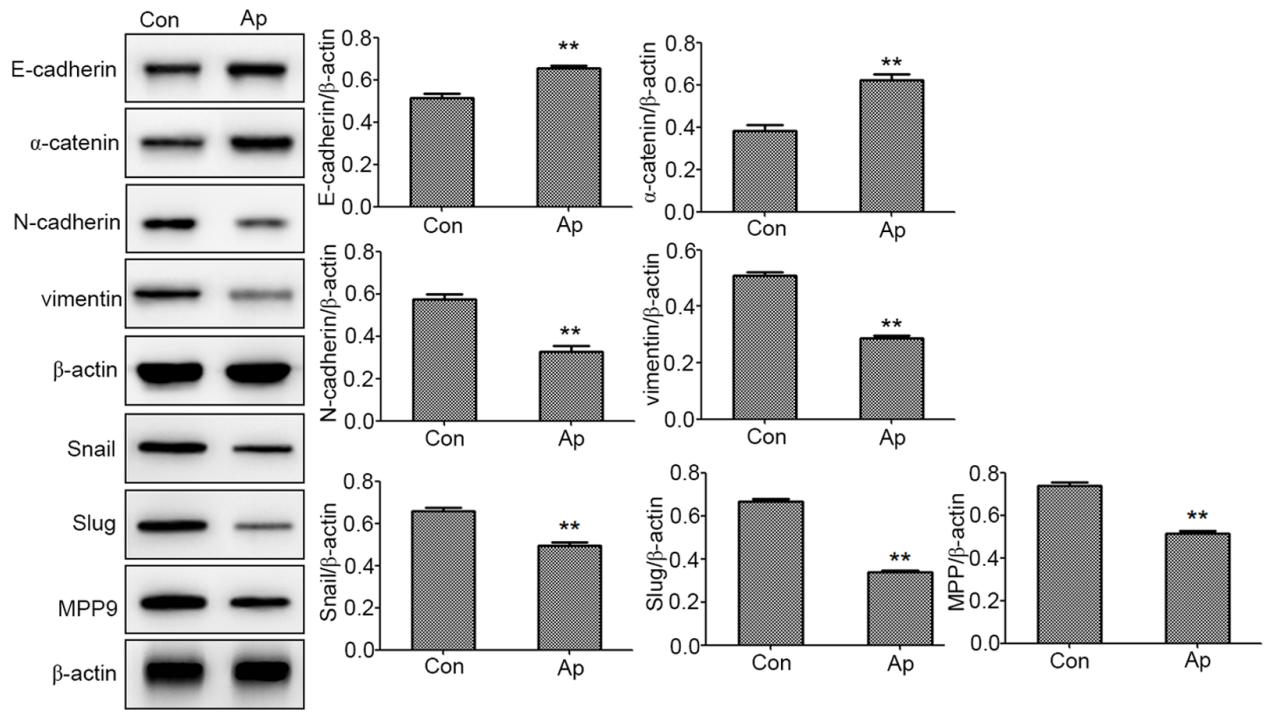

D

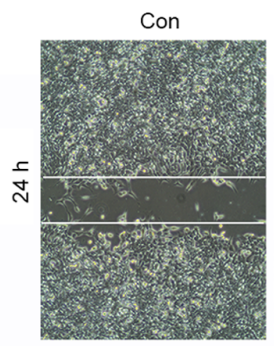

Ap

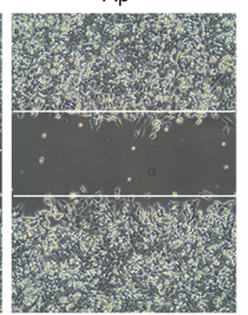

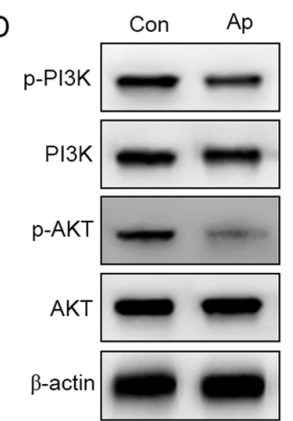

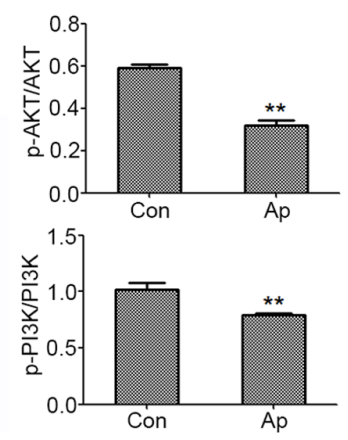

Figure 2. Apatinib inhibited the migration and invasion of Hep3b cells. (A) Invasion of Hep3b cells incubation with apatinib for 24 was determined by Transwell assay (magnification, x100). (B) Migration of Hep3b cells in response to apatinib was determined by wound scratch assay at 0 and $24 \mathrm{~h}$ under microscope (magnification, x100). (C) Western blotting was used to measure the expression level of EMT-related proteins E-cadherin, $\alpha$-catenin, N-cadherin, Vimentin, Snail, Slug and MMP9 (D) Western blotting was used to measure the expression level of p-PI3K, PI3K, p-AKT and AKT. Values are shown as mean \pm standard deviation $(\mathrm{n}=3) ;{ }^{* *} \mathrm{P}<0.01$ vs. Con group. EMT, epithelial-mesenchymal transition; $\mathrm{p}-$, phosphorylated; Con, control; Ap, apatinib.

A

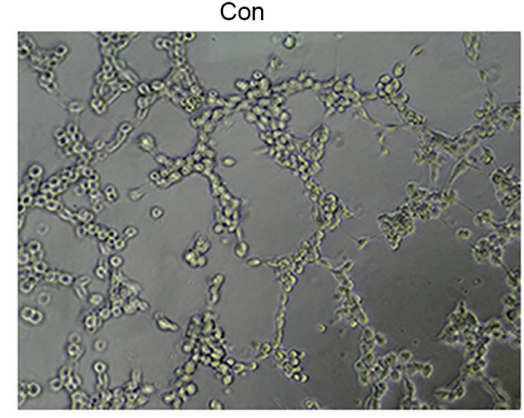

Ap

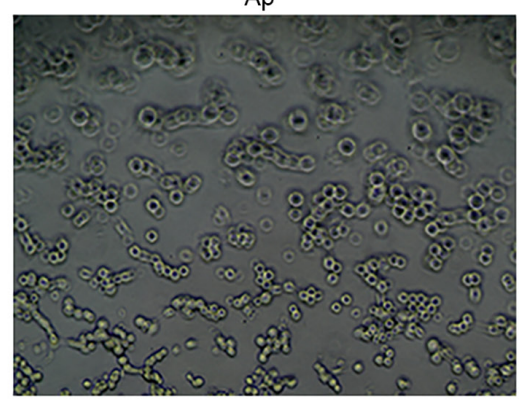

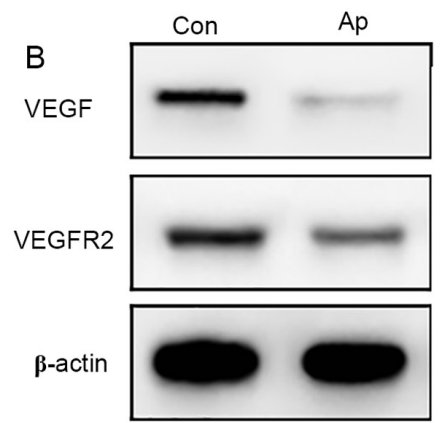
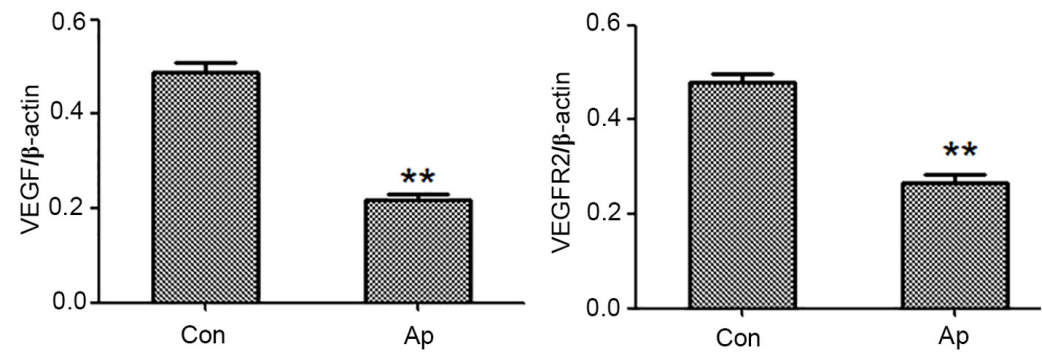

Figure 3. Apatinib inhibited the tubule formation of HUVECs. (A) Effects Hep3b cells-CM on HUVEC tube formation on Matrigel after $24 \mathrm{~h}$ were recorded with a bright field microscope (magnification, x100). (B) Western blotting was used to measure the protein expression level of VEGF and VEGFR2 in Hep3b cells. Values are shown as mean \pm standard deviation $(\mathrm{n}=3) ;{ }^{* * *} \mathrm{P}<0.01$ vs. Con group. HUVECs, human umbilical vein endothelial cells; Con, control; Ap, apatinib. 

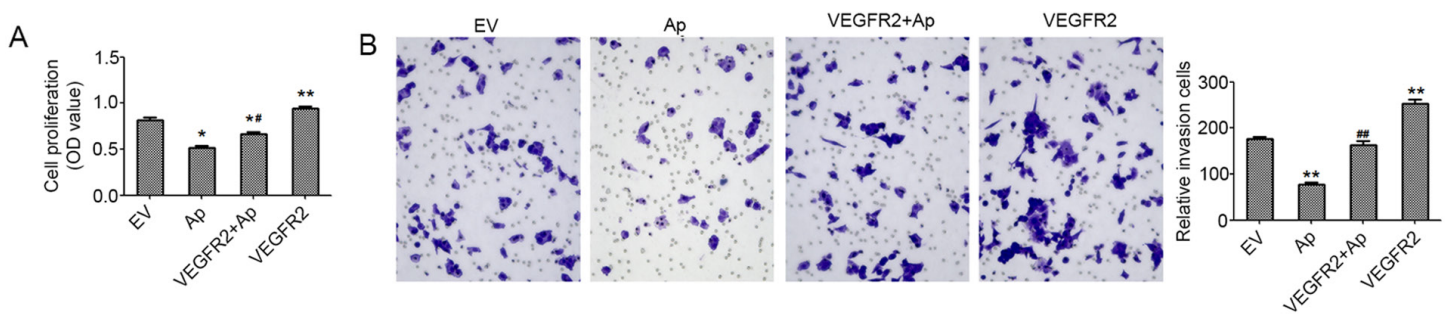

VEGFR2+AP
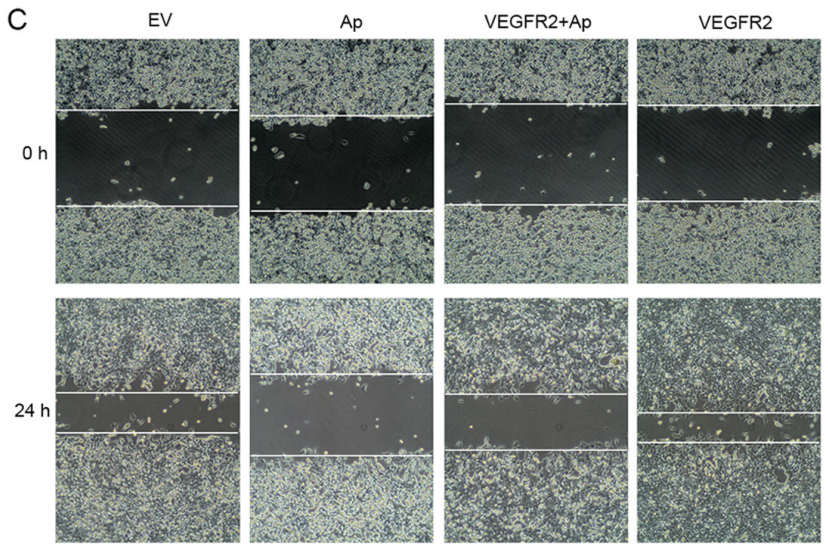

$\mathrm{E}$
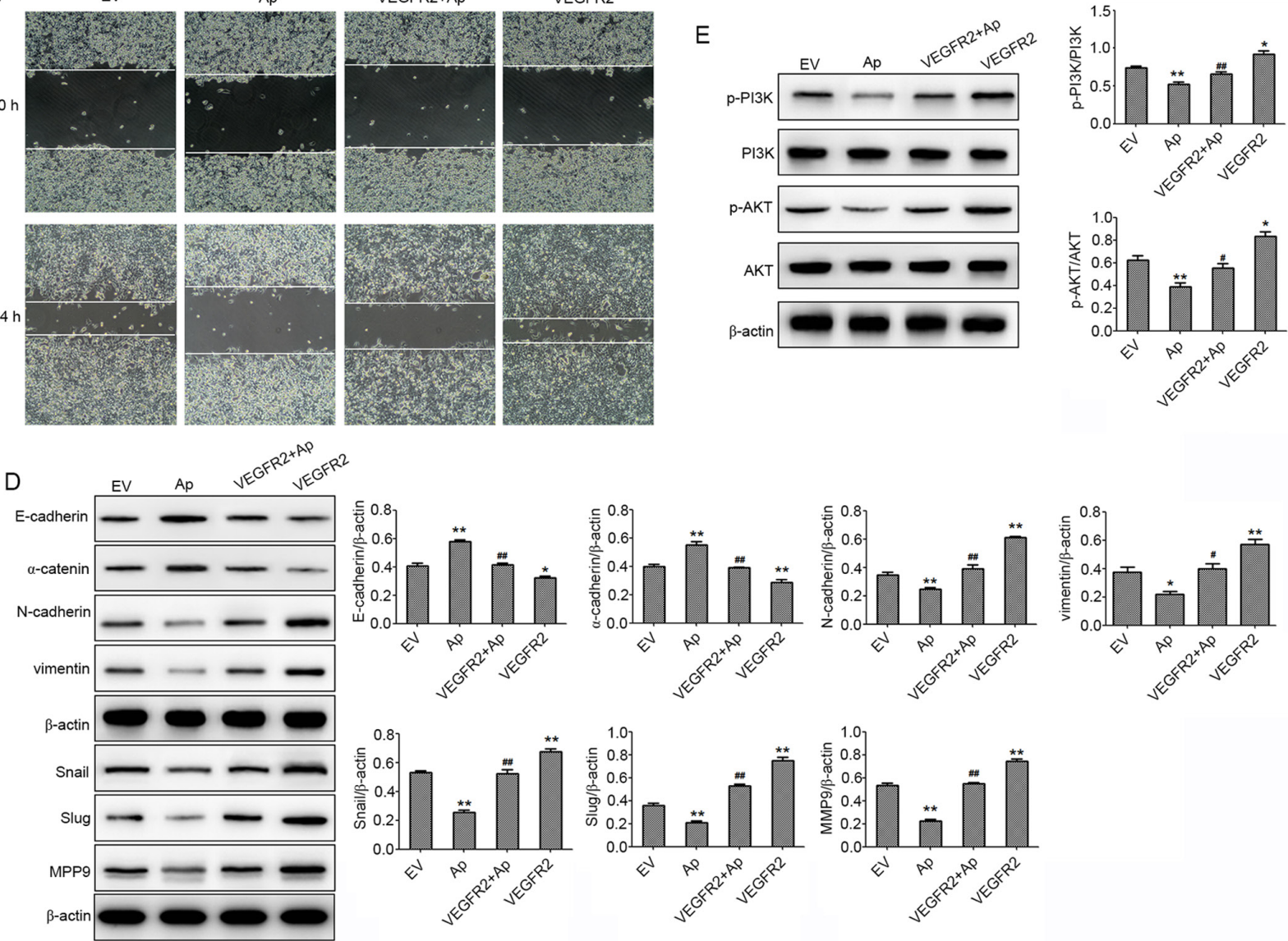

Figure 4. VEGFR2 overexpression suppresses the inhibitory effect of apatinib on Hep3b cell proliferation, migration and invasion. (A) The proliferation of Hep3b cells were detected by MTT assay. (B) Invasion of Hep3b cells was determined by Transwell assay (magnification, x100). (C) Migration of Hep3b cells was determined by wound scratch assay at 0 and $24 \mathrm{~h}$ under microscope (magnification, $\mathrm{x} 100$ ). (D) Western blotting was used to measure the expression level of EMT-related proteins E-cadherin, $\alpha$-catenin, N-cadherin, Vimentin, Snail, Slug and MMP9. (E) Western blotting was used to measure the expression level of p-PI3K, PI3K, p-AKT and AKT. Values are shown as mean \pm standard deviation $(\mathrm{n}=3) ;{ }^{*} \mathrm{P}<0.05$ or ${ }^{* *} \mathrm{P}<0.01 \mathrm{vs}$. EV group; ${ }^{\#} \mathrm{P}<0.05$ or ${ }^{\# \#} \mathrm{P}<0.01$ vs. Ap group. EMT, epithelial-mesenchymal transition; p-, phosphorylated; EV, empty vector; Ap, apatinib.

independent prognostic factors, such as vascular invasion, metastasis stage and tumor differentiation $(30,31)$. The activation of the PI3K/AKT pathway is reported to enhance the invasion and metastasis of HCC cells (32). The present study found that apatinib treatment reduced the phosphorylation of PI3K and AKT in Hep3b cells, indicating that a strong inhibitory effect of apatinib on Hep3b cell migration and invasion is associated with the inhibition of the PI3K/AKT signaling pathway. Similar results have found in NSCLC cells, in which the apatinib treatment synergistically reduced proliferation and inhibited the migration and invasion of NSCLC cells (33).

A previous study reported that apatinib treatment significantly attenuated macrophage infiltration and EMT of lung tissue (20). EMT is a fundamental process during which tumor cells acquire the capacity of migration and invasion (34). It is mainly characterized by the downregulation of cell adhesion molecules [including E-cadherin, $\alpha$-catenin and zonula occludens-1 (ZO-1)], transformation of the cytokeratin cytoskeleton into vimentin and morphological characteristics of mesenchymal cells (35). E-cadherin, ZO-1 and $\alpha$-catenin are necessary to form stable adherens junctions (36). During the EMT process, epithelial cells lose E-cadherin and transform into spindle shaped mesenchymal cells by acquiring $\mathrm{N}$-cadherin. Vimentin and fibronectin are mesenchymal markers that are overexpressed in cancer cells, demonstrating that these factors promote tumor growth, metastasis and recurrence (37). Zheng et al (27) suggested that apatinib inhibits the migration and invasion of osteosarcoma by targeting STAT3 pathway to inhibit EMT. The present study found that the apatinib treatment increased the expressions of the epithelial hallmarks E-cadherin and $\alpha$-catenin and decreased the expressions of the mesenchymal hallmarks N-cadherin, Vimentinin, Snail, Slug and MMP9 in Hep3b cells. These results indicated 
A

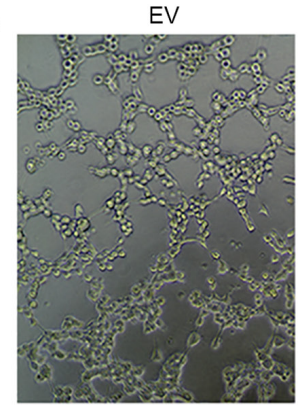

Ap

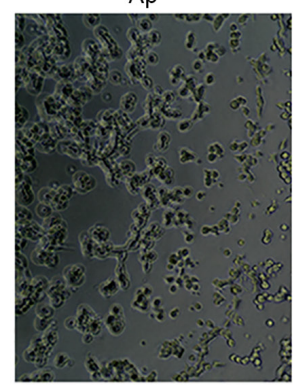

VEGFR2+Ap

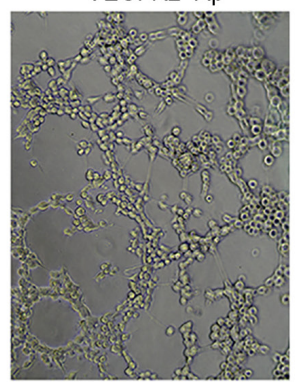

VEGFR2

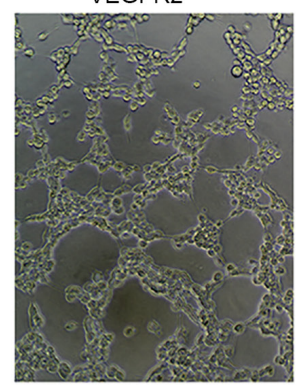

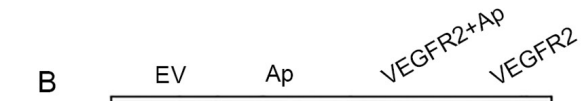
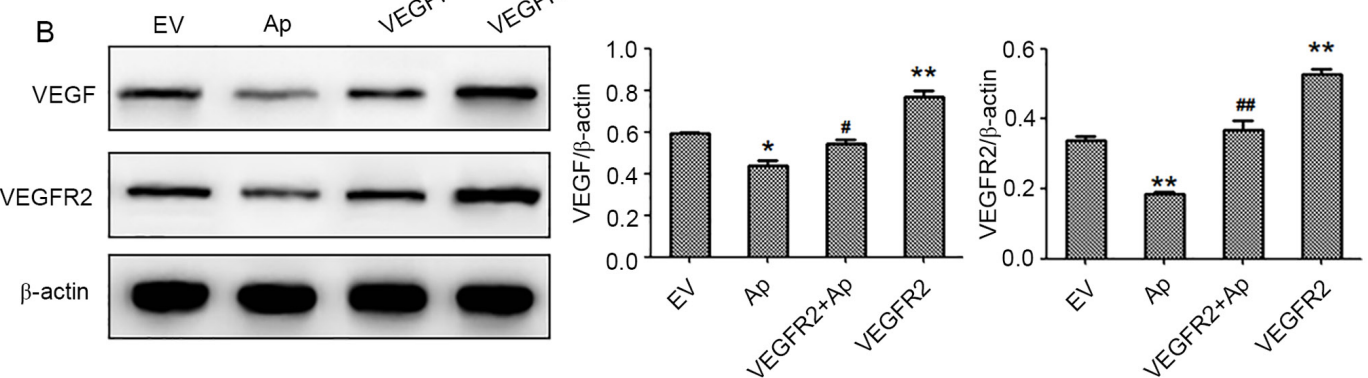

Figure 5. VEGFR2 overexpression suppresses the inhibitory effect of apatinib on the angiogenesis of HUVEC cells. (A) Effects Hep3b cells-CM on HUVEC tube formation on Matrigel after $24 \mathrm{~h}$ were recorded with a bright field microscope (magnification, $\mathrm{x} 100$ ). (B) Western blotting was used to measure the protein expression level of VEGF and VEGFR2 in Hep3b cells. Values are shown as mean \pm standard deviation $(\mathrm{n}=3) ;{ }^{*} \mathrm{P}<0.05$ or ${ }^{* *} \mathrm{P}<0.01$ vs. EV group; ${ }^{\#} \mathrm{P}<0.05$ or ${ }^{\# \prime} \mathrm{P}<0.01$ vs. Ap group. HUVECs, human umbilical vein endothelial cells; CM, conditioned media; EV, empty vector; Ap, apatinib.

that apatinib attenuates the migration and invasion of Hep3b cells by regulating EMT.

HCC is a typical hypervascular tumor with a high VEGFR expression (38). VEGF promotes angiogenesis by inducing proliferation and migration of endothelial cells (39). Tumor angiogenesis provides essential growth that requires nutrients and oxygen for tumor occurrence, development and metastasis and which are closely associated with tumor stage and prognosis (40-42). Therefore, studies have been conducted to explore agents that target the VEGF axis in advanced HCC (43-45). It has been reported that apatinib effectively inhibits the proliferation, migration and tube formation of HUVEC by blocking the VEGF axis (14). Chen et al (21) reported that the inhibitory effects of apatinib on EMT and tumorigenesis may be associated with the downregulation of the expression levels of MMP2/9, VEGF and VEGFR2 in HCC. Similarly, apatinib had an inhibitory effect on the VEGF/VEGFR pathway, Hep3b cell migration, invasion and tube formation in the present study (46). A previous study reported that the $\mathrm{PI} 3 \mathrm{~K} / \mathrm{Akt} / \mathrm{NF}-\kappa \mathrm{B}$ pathway can regulate the invasion of carcinoma cells via upregulation of VEGF, indicating that VEGF is the downstream target of the PI3K/AKT pathway in regulating cancer invasion and metastasis (47). In addition, apatinib treatment inhibits tumor growth and angiogenesis in anaplastic thyroid carcinoma via suppression of the AKT pathway way (48). The present study found that apatinib inhibited the expressions of VEGF and VEGFR2 and reduced the activation of PI3K/AKT. Conversely, the VEGFR2 overexpression markedly increased the activation of the PI3K/AKT pathway. These results indicated that the anti-angiogenic effect of apatinib in Hep3b cells may be mediated, at least in part, by preventing the VEGF expression and subsequent decrease in PI3K/AKT pathway activation.
In conclusion, the data presented in the current study revealed that apatinib suppressed the proliferation, migration and invasion of Hep3b cells by inhibiting the PI3K-AKT pathway-mediated EMT. In addition, it suppressed the expression and activity of pro-angiogenic factors VEGF and VEGFR-2, which may also involve the activation of the downstream PI3K-AKT signaling pathway, resulting in the inhibition of angiogenesis. These findings indicated that apatinib has a great potential for use as an antitumor agent in patients with HCC by inhibiting cell migration, invasion and angiogenesis by blocking the VEGF and PI3K/AKT pathways. A further study on the effect apatinib effect on other liver cancer cell lines and in vivo animal experiment will be of great interest to fully elucidate the underlying signaling pathways involved in its anti-cancer effect.

\section{Acknowledgements}

Not applicable.

\section{Funding}

This work was supported by the Qingdao Health Science and Technology Project (grant no. 2016-WJZD076).

\section{Availability of data and materials}

The datasets used and/or analyzed during the current study are available from the corresponding author on reasonable request.

\section{Authors' contributions}

JS, ZG and YZ designed the experiments. JS, CS and ML performed most of the experiments. JS drafted the manuscript. 
ZG contributed to the analysis and interpretation of the data. YZ modified the manuscript. All authors read and approved the final manuscript.

\section{Ethics approval and consent to participate}

Not applicable.

\section{Patient consent for publication}

Not applicable.

\section{Competing interests}

The authors declare that they have no competing interests.

\section{References}

1. Forner A, Reig M and Bruix J: Hepatocellular carcinoma. Lancet 391: 1301-1314, 2018.

2. Yang N, Ekanem NR, Sakyi CA and Ray SD: Hepatocellular carcinoma and microRNA: New perspectives on therapeutics and diagnostics. Adv Drug Deliv Rev 81: 62-74, 2015.

3. Bruix J, Gores GJ and Mazzaferro V: Hepatocellular carcinoma: Clinical frontiers and perspectives. Gut 63: 844-855, 2014.

4. Kalluri R and Weinberg RA: The basics of epithelial-mesenchymal transition. J Clin Invest 119: 1420-1428, 2009.

5. Radisky DC: Epithelial-mesenchymal transition. J Cell Sci 118: 4325-4326, 2005.

6. Wang SH, Wu XC, Zhang MD, Weng MZ, Zhou D and Quan ZW: Upregulation of $\mathrm{H} 19$ indicates a poor prognosis in gallbladder carcinoma and promotes epithelial-mesenchymal transition. Am J Cancer Res 6: 15-26, 2015.

7. Huang Q, Han J, Fan J, Duan L, Guo M, Lv Z, Hu G, Chen L, Wu F, Tao X, et al: IL-17 induces EMT via Stat 3 in lung adenocarcinoma. Am J Cancer Res 6: 440-451, 2016.

8. Li M, Zhang B, Sun B, Wang X, Ban X, Sun T, Liu Z and Zhao X: A novel function for vimentin: The potential biomarker for predicting melanoma hematogenous metastasis. J Exp Clin Cancer Res 29: 109, 2010.

9. Han J, Wang F, Lan Y, Wang J, Nie C, Liang Y, Song R, Zheng T, Pan S, Pei T, et al: KIFC1 regulated by miR-532-3p promotes epithelial-to-mesenchymal transition and metastasis of hepatocellular carcinoma via gankyrin/AKT signaling. Oncogene 38: 406-420, 2019

10. Yang X, Zhang XF, Lu X, Jia HL, Liang L, Dong QZ, Ye QH and Qin LX: MicroRNA-26a suppresses angiogenesis in human hepatocellular carcinoma by targeting hepatocyte growth factor-cMet pathway. Hepatology 59: 1874-1885, 2014.

11. Liu Y, Qiao Y, Hu C, Liu L, Zhou L, Liu B, Chen H and Jiang X VEGFR2 inhibition by RNA interference affects cell proliferation, migration, invasion, and response to radiation in Calucells. Clin Transl Oncol 18: 212-219, 2016.

12. Minata M, Harada KH, Kudo M, Ikai I and Nishida N: The prognostic value of vascular endothelial growth factor in hepatocellular carcinoma for predicting metastasis after curative resection. Oncology 84 (Suppl 1): 75-81, 2013.

13. Chatterjee S, Heukamp LC, Siobal M, Schöttle J, Wieczorek C, Peifer M, Frasca D, Koker M, König K, Meder L, et al: Tumor VEGF:VEGFR2 autocrine feed-forward loop triggers angiogenesis in lung cancer. J Clin Invest 123: 1732-1740, 2013.

14. Tian S, Quan H, Xie C, Guo H, Lü F, Xu Y, Li J and Lou L: YN968D1 is a novel and selective inhibitor of vascular endothelial growth factor receptor-2 tyrosine kinase with potent activity in vitro and in vivo. Cancer Sci 102: 1374-1380, 2011.

15. Li F, Liao Z, Zhao J, Zhao G, Li X, Du X, Yang Y and Yang J: Efficacy and safety of Apatinib in stage IV sarcomas: Experience of a major sarcoma center in China. Oncotarget 8: 64471-64480, 2017.

16. Li J, Qin S, Xu J, Xiong J, Wu C, Bai Y, Liu W, Tong J, Liu $\mathrm{Y}, \mathrm{Xu} \mathrm{R}$, et al: Randomized, double-blind, placebo-controlled phase III trial of apatinib in patients with chemotherapy-refractory advanced or metastatic adenocarcinoma of the stomach or gastroesophageal junction. J Clin Oncol 34: 1448-1454, 2016.
17. Hu X, Zhang J, Xu B, Jiang Z, Ragaz J, Tong Z, Zhang Q, Wang X, Feng J, Pang D, et al: Multicenter phase II study of apatinib, a novel VEGFR inhibitor in heavily pretreated patients with metastatic triple-negative breast cancer. Int J Cancer 135: 1961-1969, 2014.

18. Li J, Qin S, Xu J, Guo W, Xiong J, Bai Y, Sun G, Yang Y, Wang L, Xu N, et al: Apatinib for chemotherapy-refractory advanced metastatic gastric cancer: Results from a randomized, placebo-controlled, parallel-arm, phase II trial. J Clin Oncol 31: 3219-3225, 2013

19. Qin S, Bai Y, Ouyang X, Cheng Y, Li J, Xu J, Liang J, Li Q, Wu W and Liu W: Apatinib for patients with advanced hepatocellular carcinoma: A randomised, open-label, multicentre, phase II clinical trial. Lin Chuang Zhong Liu Xue Za Zhi 22: 1057-1065, 2017 (In Chinese).

20. Liu S, Su L, Mu X, Shi Y, Zhang A and Ge X: Apatinib inhibits macrophage-mediated epithelial - mesenchymal transition in lung cancer. RSC Advances 8: 21451-21459, 2018.

21. Chen Y, Chen X, Ding X and Wang Y: Afatinib, an EGFR inhibitor, decreases EMT and tumorigenesis of Huh 7 cells by regulating the ERK VEGF/MMP9 signaling pathway. Mol Med Rep 20: 3317-3325, 2019.

22. Zhang Y, Wang SJ, Han ZH, Li YQ, Xue JH, Gao DF, Wu XS and Wang CX: PI3K/AKT signaling pathway plays a role in enhancement of eNOS activity by recombinant human angiotensin converting enzyme 2 in human umbilical vein endothelial cells. Int J Clin Exp Pathol 7: 8112-8117, 2014.

23. Xu W, Yang Z and Lu N: A new role for the PI3K/Akt signaling pathway in the epithelial-mesenchymal transition. Cell Adhes Migr 9: 317-324, 2015

24. Yu X, Zheng Y, Zhu X, Gao X, Wang C, Sheng Y, Cheng W, Qin L, Ren N, Jia H, et al: Osteopontin promotes hepatocellular carcinoma progression via the PI3K/AKT/Twist signaling pathway. Oncol Lett 16: 5299-5308, 2018.

25. Scott AJ, Messersmith WA and Jimeno A: Apatinib: A promising oral antiangiogenic agent in the treatment of multiple solid tumors. Drugs Today (Barc) 51: 223-229, 2015.

26. Liu K, Ren T, Huang Y, Sun K, Bao X, Wang S, Zheng B and Guo W: Apatinib promotes autophagy and apoptosis through VEGFR2/STAT3/BCL-2 signaling in osteosarcoma. Cell Death Dis 8: e3015, 2017.

27. Zheng B, Ren T, Huang Y and Guo W: Apatinib inhibits migration and invasion as well as PD-L1 expression in osteosarcoma by targeting STAT3. Biochem Biophys Res Commun 495: 1695-1701, 2018.

28. Huang M, Huang B, Li G and Zeng S: Apatinib affect VEGFmediated cell proliferation, migration, invasion via blocking VEGFR2/RAF/MEK/ERK and PI3K/AKT pathways in cholangiocarcinoma cell. BMC Gastroenterol 18: 169, 2018.

29. Saxena NK, Sharma D, Ding X, Lin S, Marra F, Merlin D and Anania FA: Concomitant activation of the JAK/STAT, PI3K/AKT, and ERK signaling is involved in leptin-mediated promotion of invasion and migration of hepatocellular carcinoma cells. Cancer Res 67: 2497-2507, 2007.

30. Zhou Q, Lui VW and Yeo W: Targeting the PI3K/Akt/mTOR pathway in hepatocellular carcinoma. Future Oncol 7: 1149-1167, 2011.

31. Fathi Maroufi N, Rashidi MR, Vahedian V, Akbarzadeh M, Fattahi A and Nouri M: Therapeutic potentials of Apatinib in cancer treatment: Possible mechanisms and clinical relevance. Life Sci 241: 117106, 2020.

32. Lou L, Ye W, Chen Y, Wu S, Jin L, He J, Tao X, Zhu J, Chen X, Deng A, et al: Ardipusilloside inhibits survival, invasion and metastasis of human hepatocellular carcinoma cells. Phytomedicine 19: 603-608, 2012.

33. Liao X, Tao L, Guo W, Wu ZX, Du H, Wang J, Zhang J, Chen H, Chen ZS, Lin L, et al: Combination of Cordycepin and Apatinib Synergistically Inhibits NSCLC Cells by Down-Regulating VEGF/PI3K/Akt Signaling Pathway. Front Oncol 10: 1732, 2020.

34. Chen T, You Y, Jiang H and Wang ZZ: Epithelial-mesenchymal transition (EMT): A biological process in the development, stem cell differentiation, and tumorigenesis. J Cell Physiol 232: 3261-3272, 2017.

35. Song L, Li XX, Liu XY, Wang Z, Yu Y, Shi M, Jiang B and He XP: EFEMP2 suppresses the invasion of lung cancer cells by inhibiting epithelial-mesenchymal transition (EMT) and down-regulating MMPs. OncoTargets Ther 13: 1375-1396, 2020.

36. Piao HL, Yuan Y, Wang M, Sun Y, Liang H and Ma L: $\alpha$-catenin acts as a tumour suppressor in E-cadherin-negative basal-like breast cancer by inhibiting NF- $\mathrm{KB}$ signalling. Nat Cell Biol 16: $245-254,2014$ 
37. Niknami Z, Eslamifar A, Emamirazavi A, Ebrahimi A and Shirkoohi R: The association of vimentin and fibronectin gene expression with epithelial-mesenchymal transition and tumor malignancy in colorectal carcinoma. EXCLI J 16: 1009-1017, 2017.

38. Yamaguchi R, Yano H, Nakashima Y, Ogasawara S, Higaki K, Akiba J, Hicklin DJ and Kojiro M: Expression and localization of vascular endothelial growth factor receptors in human hepatocellular carcinoma and non-HCC tissues. Oncol Rep 7: 725-729, 2000.

39. Chen H, Shen YF, Gong F, Yang GH, Jiang YQ and Zhang R: Expression of VEGF and its effect on cell proliferation in patients with chronic myeloid leukemia. Eur Rev Med Pharmacol Sci 19: $3569-3573,2015$

40. Podar K and Anderson KC: Inhibition of VEGF signaling pathways in multiple myeloma and other malignancies. Cell Cycle 6: 538-542, 2007.

41. Lin D and Wu J: Hypoxia inducible factor in hepatocellular carcinoma: A therapeutic target. World J Gastroenterol 21: 12171-12178, 2015

42. Song P, Gao J, Inagaki Y, Kokudo N, Hasegawa K, Sugawara Y and Tang W: Biomarkers: Evaluation of screening for and early diagnosis of hepatocellular carcinoma in Japan and China. Liver Cancer 2: 31-39, 2013

43. Buijs N, Oosterink JE, Jessup M, Schierbeek H, Stolz DB, Houdijk AP, Geller DA and van Leeuwen PA: A new key player in VEGF-dependent angiogenesis in human hepatocellular carcinoma: Dimethylarginine dimethylaminohydrolase 1 . Angiogenesis 20 557-565, 2017.
44. Bhoori S and Mazzaferro V: Combined immunotherapy and VEGF-antagonist in hepatocellular carcinoma: A step forward. Lancet Oncol 21: 740-741, 2020.

45. Yao Y, Wang T, Liu Y and Zhang N: Co-delivery of sorafenib and VEGF-siRNA via pH-sensitive liposomes for the synergistic treatment of hepatocellular carcinoma. Artif Cells Nanomed Biotechnol 47: 1374-1383, 2019.

46. Yang C and Qin S: Apatinib targets both tumor and endothelial cells in hepatocellular carcinoma. Cancer Med 7: 4570-4583, 2018.

47. Shen K, Ji L, Gong C, Ma Y, Yang L, Fan Y, Hou M and Wang Z: Notoginsenoside Ftl promotes angiogenesis via HIF-1 $\alpha$ mediated VEGF secretion and the regulation of PI3K/AKT and Raf/MEK ERK signaling pathways. Biochem Pharmacol 84: 784-792, 2012. 48. Jin Z, Cheng X, Feng H, Kuang J, Yang W, Peng C, Shen B and Qiu W: Apatinib inhibits angiogenesis via suppressing Akt/GSK3 $\beta / A N G$ signaling pathway in anaplastic thyroid cancer. Cell Physiol Biochem 44: 1471-1484, 2017.

(7) (9) This work is licensed under a Creative Commons

C. International (CC BY-NC-ND 4.0) License. 